\title{
Do People Make Strategic Commitments? Experimental Evidence on Strategic Information Avoidance
}

\author{
Anders U. Poulsen* and Michael W. M. Roos ${ }^{\dagger}$
}

March 10, 2010

\begin{abstract}
Game theory predicts that players make strategic commitments that may appear counter-intuitive. We conducted an experiment to see if people make a counter-intuitive but strategically optimal decision to avoid information. The experiment is based on a sequential Nash demand game in which a responding player can commit ahead of the game not to see what a proposing player demanded. Our data show that subjects do, but only after substantial time, learn to make the optimal strategic commitment. We find only weak evidence of physical timing effects.
\end{abstract}

Keywords: Strategic commitment; commitment; bargaining; strategic value of information; physical timing effects; endogenous timing; experiment.

JEL Classification: C72; C78; C90; C92; D63; D80.

\section{Introduction}

A crucial insight from game theory is that a player involved in an interactive situation can gain from making a strategic commitment. ${ }^{1}$ Well-known examples of strategic

\footnotetext{
*Corresponding author. School of Economics, University of East Anglia, Norwich NR4 7TJ, United Kingdom. E-mail: a.poulsen@uea.ac.uk. Poulsen is member of the Centre for Behavioural and Experimental Social Science at the University of East Anglia, and affiliate researcher at the Centre for Experimental Economics at University of Copenhagen. We thank the Editor Jordi Brandts and two reviewers whose very valuable comments and suggestions significantly improved the analysis of the data and the exposition of the results. All errors are of course ours. We wish to thank Niels Bruhn Christensen for invaluable help with the design and the programming. Thanks to Chaim Fershtman, Werner Güth, Hans-Theo Normann, and to the GATE and UEA seminar audiences for their comments. Toke Fosgaard, Mads Harmsen, and Frederik Øvlisen from the LEE lab in Copenhagen gave professional lab assistance. Financial support from the Danish Social Science Research Council (Poulsen) is gratefully acknowledged.

†Fakultät für Wirtschaftswissenschaft, Ruhr-Universität Bochum, D-44780 Bochum, Germany.

${ }^{1}$ Schelling $(1960,160)$ uses the term, "strategic move".
} 
commitments are moving before someone else to get a first-mover advantage (Bagwell, 1995, Huck and Müller, 2000, and Schelling, 1960); signing contracts with thirdparties (Aghion and Bolton, 1985, and Bensaid and Gary-Bobo, 1993); burning money (Ben-Porath and Dekel, 1992, van Damme, 1989, Huck and Müller, 2005); strategic delegation (Fershtman and Gneezy, 2001, Fershtman and Kalai, 1997, and Schelling, 1960); changing the information structure (Hauk and Hurkens, 2001, Hurkens and Vulkan, 2006, and Schelling, 1960) or controlling the flow of information (Brocas and Carrillo, 2007).

These important game-theoretic results about strategic commitments lead naturally to the following question: Do players in practice understand the usefulness of making such strategic commitments? This, we believe, is a non-trivial issue. The strategic commitments mentioned above involve a deliberate restriction of one's freedom of action, a reduction of one's payoffs in certain outcomes, or an avoidance of information. Such moves may appear irrational and unattractive, and players may consequently avoid them. Also, bounded rationality or cognitive biases could lead players to underestimate or completely ignore the impact of the strategic commitment on the other players' behavior. Such biases have been experimentally documented in other, mostly non-strategic, decision problems. See Loewenstein, Moore, and Weber (2006) and the references therein.

In this paper we consider games where the strategic commitment concerns information acquisition. Many important interactive economic situations involve decisions about how much information to collect. Some examples are negotiators engaging in fact finding missions before sitting down at the negotiating table, and bidders going to viewing days to get a feel for the value of the items on sale (see Hauk and Hurkens, 2001, and Hurkens and Vulkan, 2006). It is useful when considering such situations to know the extent to which players make the theoretically predicted strategic commitment since deviations from the prediction could have sizeable economic implications for the players.

To shed empirical light on these questions we conducted an experiment using a simple bargaining situation based on the Nash demand game (Nash, 1953). In our first treatment a player, the Responder, has the opportunity to make a strategic commitment of seeing or not seeing what another player, the Proposer, has demanded before the Responder makes a demand, and this strategic commitment is observed by the Proposer before he makes his own demand. We call this move strategic information avoidance. Our second treatment is the same as the first except for the fact that the Responder's strategic commitment is not observed by the Proposer. The theoretical model predicts that the Responder avoids information in the first and obtains it in the second treatment.

The crucial role of the visibility of the strategic commitment was emphasised early on in Schelling (1960). We also test a related theoretical result and intuition, namely that behavior in the situation where the Responder's strategic commitment is not observed by the Proposer is the same as in the situation where the Responder makes the strategic commitment after the Proposer has moved. This hypothesis is of some interest since experimental research have shown that the order of moves can influence behavior although game theory predicts it should not; see for example Güth, Huck, and Rapoport (1998), Weber, Camerer, and Knez (2006), and Huck and Müller 
(2005). This is referred to as 'physical timing effects' or 'virtual observability'. Finally we consider the hypothesis that when the Responder's strategic commitment is unobserved players behave in the same way as in the situation where the Responder has no commitment option available.

Other experimental papers have studied issues related to strategic commitments and information acquisition. Fischer, Güth, Müller, and Stiehler (2006) consider the case where a second mover is with a certain probability informed about the first mover's action. Fonseca, Müller, and Normann (2006) consider the choice of when to move (endogenous timing), and hence how much information to possess, in a duopoly game. Studying the ultimatum game Poulsen and Tan (2007) consider the decision of Proposers whether or not to learn the Responders' smallest acceptable offers. We discuss how our experiment relates to these contributions in Section 5 below.

The main purpose of the experiment is to see if people make the predicted strategic commitment in the various strategic environments described above. Our experimental findings can be summarized as follows: First, players learn to make the optimal strategic commitment both in the situation where it is best to be informed and in the situation where it is best to avoid information. It does however take more time for players to learn to avoid harmful information than it does for them to learn to obtain beneficial information; indeed, in the latter case most players make the optimal strategic commitment right from the start. Second, although we find some evidence of a physical timing effect in the initial periods of the experiment, it becomes insignificant over time. Finally, we find as predicted by game theory no significant behavior differences between the case where commitment is unobserved and when no commitment is available at all.

These findings can be intuitively explained when we consider the strategic reasoning that is required by Responders in on order for them to make the optimal strategic commitment. As we show below, an argument based on weak dominance implies that information is optimal for the Responder whenever his or her information decision is unobserved by the Proposer. Our data show that subjects appreciate this more or less from the outset, and that physical timing is not an important factor. On the other hand, when the Responder's information decision is observed by the Proposer, the required reasoning is more complex: the Responder must conjecture how the Proposer's demand will depend on the Responder's information decision, which in turn requires simulating the Responder's own demand reaction. Here both backward and forward induction-like reasoning is required, which seems much more complex than the weak dominance argument just described. Indeed, our data show that it takes substantial time for Responders to learn the latter kind of reasoning. Overall, the findings indicate that players make the predicted strategic commitments when they have sufficient time to understand the situation, and that the time required depends on the complexity of the required reasoning.

The rest of the paper is organized as follows. In Section 2 we develop the theoretical background and our hypotheses. Section 3 describes the experiment. Section 4 reports our findings and these are discussed and related to the existing literature in Section 5. Section 6 concludes and outlines some possible future research. The Appendix contains the proofs of the theoretical propositions. 


\section{Theoretical Predictions}

\subsection{The Games}

Our experiment is based on four games: A game where a player's strategic commitment is made and observed by the other player before the latter chooses an action (called the 'Commitment game'); a second game where the strategic commitment is made at the same time as in Commitment game but is unobserved ('Unobserved Commitment'); a third game where the strategic commitment is made after the other player has decided on an action, and hence is trivially unobserved ('No Commitment'); and, finally, a game where no strategic commitment is available ('Benchmark'). In what follows we describe these games more precisely and develop hypotheses.

There are two players, the Proposer $(\mathrm{P})$ and the Responder $(\mathrm{R})$, and a money surplus, denoted $X$. Let $\epsilon>0$ denote the smallest monetary unit. The set of feasible demands is $D=\{0, \epsilon, 2 \epsilon, \ldots, X-\epsilon, X\}$. We assume that $X$ is even, that $\epsilon$ is such that $X / 2 \in D$, and that $\epsilon<X / 2$. Denote player $i$ 's demand by $x_{i}, i=P, R$, where $x_{i} \in D$. If $x_{P}+x_{R} \leq X$, each player gets what he demanded. If $x_{P}+x_{R}>X$, each player gets zero. In our analysis we assume all players are rational and seek to maximize their expected money earnings, and that this is common knowledge.

The Benchmark Game (BM) The BM game is a sequential Nash demand game with perfect information. The Proposer first makes a demand. The Responder sees the Proposer's demand, and then the Responder makes his demand.

The Commitment Game (C) In the C game the Responder first irrevocably decides whether or not to see the demand that the Proposer will make. We refer to this as the Responder's information decision. The Proposer observes which information decision the Responder made, and then the Proposer makes his demand. The Responder then sees the Proposer's demand or not, as determined by his information decision. Finally the Responder makes his demand.

The Unobserved Commitment Game (UC) In the UC game the Responder first irrevocably decides whether or not to see the Proposer's demand, as in the C game. The Proposer does however not learn the Responder's information decision. In other words, the Proposer knows that the Responder has made some information decision (to see or not to see the Proposer's demand), but the Proposer does not know which information decision the Responder made. Then the Proposer makes a demand. After this the Responder sees the Proposer's demand or not, as determined by the Responder's information decision. Finally the Responder makes his demand.

Any difference between behavior in the $\mathrm{C}$ and $\mathrm{UC}$ game is due to the observability or otherwise of the Responder's information decision.

The No Commitment Game (NC) In the No Commitment game the Responder has no opportunity to commit to see the Proposer's demand or not before the Proposer 
makes a demand. The order of moves are: The Proposer first makes a demand. Then the Responder decides whether or not to see the Proposer's demand. Finally, the Responder makes his demand.

The UC and NC games are strategically equivalent. Any difference in behavior is solely due to a physical timing effect, as described in the papers mentioned in the Introduction.

\section{$2.2 \quad$ Equilibria}

There is a multiplicity of Nash equilibria in our bargaining games. To obtain a unique prediction we select among equilibria by making some assumptions.

Assumption 1. a. If, in the C game, the Responder decides not to see the Proposer's demand, each player demands half the surplus. b. Players do not use weakly dominated strategies, and this is common knowledge.

The first assumption can be justified as follows. Suppose in the C game the Responder decides not to see the Proposer's demand. In the subgame that follows any feasible pair of demands $\left(x_{P}, x_{R}\right)$ such that $x_{P}+x_{R}=X$ is a Nash equilibrium. The Nash equilibrium where each player demands half the surplus is the unique equilibrium that equates the players' earnings (apart from the implausible equilibrium where each player demands the entire surplus). Moreover, it is the only pure-strategy equilibrium that is symmetric, again with the exception just described in the previous parenthesis. Both these properties make the equal-split a focal equilibrium (Schelling, 1960).

The second assumption is in general a strong one. In the present context it amounts to assuming that in the UC game a Responder understands that, although he or she makes her information decision before the Proposer makes his demand, it is optimal for the Responder to see the Proposer's demand, since the Proposer cannot condition his demand on the Responder's information decision. With this being common knowledge the Proposer knows that the Responder will decide to be informed, and this selects a unique equilibrium, described below. A similar logic holds for the NC game.

Given these assumptions we obtain the following predictions for our games.

Proposition 1. a. The BM game has two pure-strategy subgame-perfect equilibria. In the first subgame-perfect equilibrium the Proposer demands $x_{P}^{*}=X$, and the Responder demands $x_{R}^{*}=X-x_{P}$ for any demand $x_{P}$ made by the Proposer. In the second subgame-perfect equilibrium the Proposer demands $x_{P}^{*}=X-\epsilon$, and the Responder demands $x_{R}^{*}=X-x_{P}$ for any demand $x_{P}<X$, and makes some demand $x_{R}^{*}>0$ for $x_{P}=X$. In any subgame-perfect equilibrium of the BM game the Responder therefore gets at most $\epsilon$ and the Proposer gets at least $X-\epsilon$.

b. Given Assumption 1a, the subgame-perfect equilibrium of the $C$ game is: The Responder decides not to see the Proposer's demand, and the Responder demands half of the surplus when it is his turn to make a demand; if the Proposer observes that the Responder decided not to see the Proposer's demand, the Proposer demands half of the surplus; and if the Proposer observes that the Responder decided to see the Proposer's demand, then one of two subgame-perfect equilibria of the ensuing subgame, which is equivalent to the BM game, is played. 
c. The unique equilibrium in weakly undominated strategies of the UC game is that the Responder decides to see the Proposer's demand, and the players' equilibrium demands are identical to those in the BM game.

d. The unique equilibrium in weakly undominated strategies of the NC game is the same as that for the UC game.

Proof: Please see the Appendix.

The result in part $\mathrm{c}$ holds for the following reason. In the UC game, given that the Proposer can not condition his demand on the Responder's information decision, the Responder strategy of seeing the Proposer's demand and then playing a best reply to the Proposer's demand weakly dominates any other Responder strategy. The domination is only weak since for any given Proposer demand the Responder strategy of not seeing the Proposer's demand and making the best reply demand earns the same payoff as the weakly dominant strategy. Given that this is common knowledge, and that players avoid weakly dominated strategies, the Proposer makes the same equilibrium demand as in the BM game. The result in part $\mathrm{d}$ is similarly derived.

\subsection{Hypotheses}

We use four treatments corresponding to our theoretical games: the Commitment $(\mathrm{C})$, the Unobserved Commitment (UC), the No Commitment (NC), and the Benchmark (BM) treatment. Based on the theoretical results in the previous section we formulate the following hypotheses.

Hypothesis 1 (information decision): The Responder avoids information about the Proposer's demand in treatment C, but obtains the information in treatment UC.

Hypothesis 2 (no physical timing effect:) The Responder makes the same information decision in treatment $\mathrm{NC}$ as in treatment $\mathrm{UC}$.

Hypothesis 3 (demands): Part A: If in treatment $\mathrm{C}$ the Responder decides not to see the Proposer's demand then each player demands half the surplus. Part B: The Proposer and Responder demands in treatment BM equal those in treatment C when the Responder decides to see the Proposer's demand. Part C: The Proposer and Responder demands in treatment BM equal the demands in treatments UC and $\mathrm{NC}$.

\section{Experimental Procedures}

The experiments took place in the spring and fall of 2006 at the Laboratory for Experimental Economics (LEE) at University of Copenhagen, Denmark. It was programmed in z-Tree (Fischbacher, 2007). The ORSEE system (Greiner, 2004) was used for recruitment. 


\subsection{Subjects}

In total 254 subjects, recruited from across the University of Copenhagen, participated in the experiment. Subjects received a show-up fee of 50 Danish Kroner (DKK), equal to about US $\$ 9$ at the time of the experiment. On average a session lasted 45 minutes. There were 4 sessions with 74 subjects for treatment BM. For the C (UC) $[\mathrm{NC}]$ treatments the sessions and subjects numbers were 4 and 72 (3 and 44) [4 and 64]. Average earnings across all treatments, including the show-up fee, was DKK 171.6, or about US $\$ 30.9$.

\subsection{Experimental Procedure}

After entering the laboratory each subject was seated in front of a computer. All computers were separated by cubicles and no verbal or visual communication between subjects took place during the experiments. Once all subjects had read the instructions, a test was distributed. When all students had answered the test questions the experimenters checked all answers. If a subject gave an incorrect answer to a question, he was asked to try again. Any questions about the instructions or the test were answered privately. Once all subjects had answered all test questions correctly, this was announced and the experiment began.

The experiment consisted of 15 periods. At the start of the experiment each subject was randomly given the Proposer or the Responder role. A subject stayed in the same role for all 15 periods. In each period one Proposer was randomly matched with a Responder. The set of feasible point demands was $\{0,1,2, \ldots, 99,100\}$ (i.e. $X=100$ and $\epsilon=1$ ). At the end of each period both players were informed about each other's demands, about the Responder's information decision, and about their own point earnings. After the last period the points a player had earned in each period were summed and converted into Danish Kroner (DKK), using the following exchange rate: 5 points is equal to DKK 1 (so 100 points equals DKK 20, or about US \$3.6). After the experiment this number of Danish Kroner was, together with the show-up fee, paid privately to each subject in a separate room.

\section{Experimental Results}

\subsection{The Responder's Information Decision}

Figure 1 shows for each of the 15 periods the percentage of Responders who decided not to see Proposer's demand in treatments C, UC, and NC.

In treatment $\mathrm{C}$ fewer than $40 \%$ of Responders initially avoid seeing the Proposer's demand. This percentage rises steadily over time, and towards the end more than $85 \%$ of the Responders avoid seeing the Proposer's demand. The average across all periods is $70.7 \%$. Behavior in the two other treatments differ markedly: In treatment NC the percentage fluctuates between 5 and $20 \%$. The average is $11.2 \%$. In treatment UC there is a initially a slight increase in the percentage of Responders who avoid 


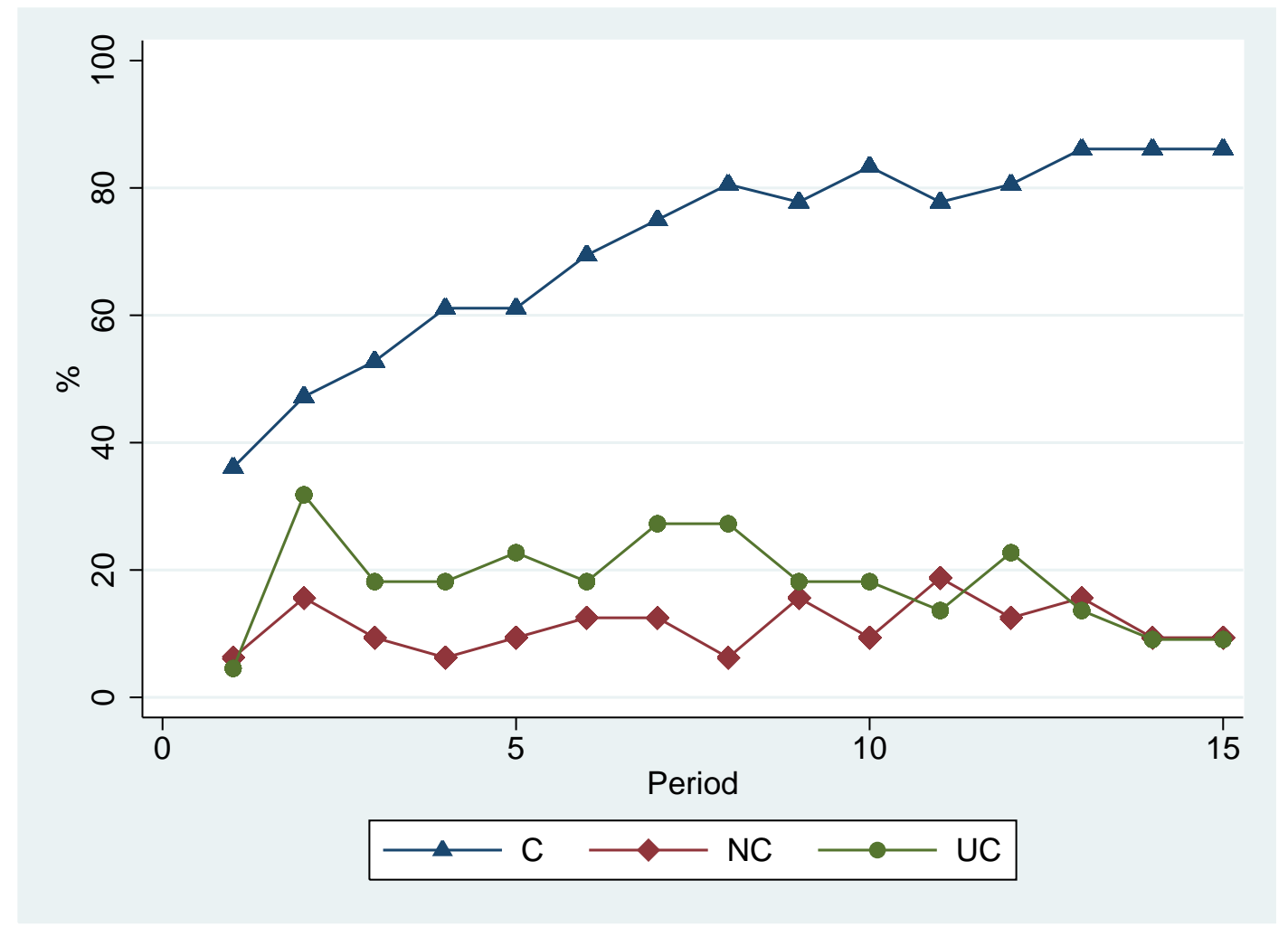

Figure 1: Percentage of Responders who decide not to see the Proposer's demand in treatment $\mathrm{C}, \mathrm{UC}$, and $\mathrm{NC}$.

information, but the percentage seems later to stabilize around $10 \%$. The average is $18.2 \%$.

To put these results on a firmer footing, and to study the dynamics of individual behavior over time, we proceed with an econometric analysis of the Responder's information decision. We first regress the fraction of Responders who decide not to observe the Proposer's demand in period $t$, denoted $N O_{t}$, on treatment dummies $T_{i}$, $i=U C, N C$ (treatment $\mathrm{C}$ is the reference group), and a linear time trend, $P E R I O D_{t}$ :

$$
N O_{t}=\beta_{C}+\beta_{U C} T_{U C}+\beta_{N C} T_{N C}+\left(\beta_{T C}+\beta_{T U C} T_{U C}+\beta_{T N C} T_{N C}\right) P E R I O D_{t}+\varepsilon_{t}
$$

This specification allows us to compare treatments $\mathrm{UC}$ and $\mathrm{NC}$ with the baseline $\mathrm{C}$ treatment. ${ }^{2}$

The results of estimating (1) are summarized in Table 1. The intercept $\beta_{C}$, which measures the initial fraction of Responders that make the predicted strategic commit-

\footnotetext{
${ }^{2}$ Two econometric problems arise when we want to estimate this model with data pooled over time and across sessions. First, there can be session-specific effects, due to the repeated interaction of the same individuals within a session. In order to control for such potential session effects, we correct for intra-group correlation (such correlation would otherwise violate the assumption of independent observations) by using the clustered sandwich estimator for the standard errors. Second, if some dynamic effects are not captured by the linear time trend there will be autocorrelation in the error term. We deal with this potential problem by assuming first-order autocorrelation in the error term and using the Prais-Winston estimator to estimate the model.
} 


\begin{tabular}{llllllllllll}
\hline \hline & $\beta_{C}$ & $\beta_{U C}$ & $\beta_{N C}$ & $\beta_{T C}$ & $\beta_{T U C}$ & $\beta_{T N C}$ & $\rho$ & $R^{2}$ & $D W_{o}$ & $D W_{t}$ & $\#$ \\
\hline coeff & .41 & -.27 & -.33 & .035 & -.035 & -.033 & .69 & .50 & .56 & 2.41 & 165 \\
$\sigma$ & .10 & .13 & .11 & .007 & .007 & .007 & & & & & \\
$\mathrm{p}$ & .003 & .071 & .015 & .000 & .000 & .001 & & & & & \\
\hline
\end{tabular}

Table 1: Responders' information decision over time and across treatments. Estimation of model (1). $\rho$ denotes the coefficient of the first-order autoregressive error term; $D W_{o}$ is the Durbin-Watson statistic of the original model with $\rho=0 ; D W_{t}$ is the Durbin Watson statistic for the transformed model controlling for autocorrelation; $\sigma$ are robust standard errors adjusted for cluster effects.

ment of not seeing the Proposer's demand in the C treatment, is significantly larger than zero but also significantly smaller than one (Wald test, $p=0.0002$ ). This means that at the beginning of the experiment a large fraction of Responders do not make the predicted information decision in treatment $\mathrm{C}$. The trend coefficient $\beta_{T C}$ is positive, so more and more Responders make the predicted information decision over time. However, 15 periods are not enough for every subject to learn the strategic move, since the trend prediction, $\beta_{C}+15 \beta_{T C}=0.935$, is significantly smaller than one (Wald test, $p=0.0026)$.

In treatment $\mathrm{UC}$ the initial fraction of information avoiding Responders is much lower than in treatment $\mathrm{C}$. In fact, the intercept for treatment $\mathrm{UC}, \beta_{C}+\beta_{U C}=$ 0.14 , is not statistically different from zero (Wald test, $p=0.1327$ ). This means that on average Responders in treatment UC make the commitment decision that is theoretically predicted. Moreover, this behavior does not change over time as there is no significant trend $\left(\beta_{T C}+\beta_{T U C}=0\right.$, Wald test, $\left.p=0.638\right)$.

Studying the aggregate proportion of Responders who make the predicted strategic commitment tells us little about the nature of any learning. Although an adjustment trend is detected in the aggregate data, individual subjects could switch back and forth between the two information decisions, which would indicate that they are not certain about the optimal behavior. To test for learning, we estimate a panel probit model with subject random effects for the probability that Responder $i$ in period $t$ decides not to observe the Proposer's demand as a function of time. Denoting the decision by Responder $i$ not to observe the Proposer's demand at time $t$ by $n o_{i t}$, the following panel probit model is a straightforward test for individual learning:

$$
P\left(n o_{i t}=1 \mid \mathbf{x}_{i t}\right)=G\left(\gamma_{0}+\gamma_{n o} n o_{i t-1}+\gamma_{T} P E R I O D_{t}\right)
$$

where $G(\cdot)$ is the standard normal cumulative distribution function and $x_{i t}$ stands for the independent variables (time and previous information decision). If individuals learn, we should expect $\gamma_{n o}>0$ and $\gamma_{T}>0$.

A potential problem with this specification is that the individual observations may not be independent. ${ }^{3}$ We deal with this by explicitly modeling the potential source of dependence. The independence assumption can be wrong if Responders use past experience to update their beliefs about how Proposers respond to the Responders' information decision. If Responders change their beliefs and their information decision

\footnotetext{
${ }^{3}$ We thank an anonymous reviewer for stressing this point.
} 
in response to their past earnings experience, this is equivalent to saying that they learn to make the predicted information decision. If we include a plausible measure of past experience with each information decision, we can capture the presence of learning, and this should ensure independent errors.

For each period $t$ we compute the average payoff that Responder $i$ received after choosing to observe the Proposer's demand:

$$
\bar{\pi}_{i t}^{o}=\frac{\sum_{\tau=1}^{t} o_{i \tau} \pi_{i \tau}}{\sum_{\tau=1}^{t} o_{i \tau}},
$$

where $o_{i \tau}$ is an indicator function equal to 1 if the Responder chose to observe the Proposer's demand in period $\tau$ and 0 otherwise. Analogously, we define $\bar{\pi}_{i t}^{n o}$ as the average payoff of the Responder after choosing not to observe the demand of the Proposer. Both measures capture the earnings feedback the Responder receives after having made the information decision. We therefore estimate an alternative random effects panel probit model for the Responders in treatment C:

$$
P\left(n o_{i t}=1 \mid \mathbf{x}_{i t}\right)=G\left(\delta_{0}+\delta_{T} P E R I O D_{t}+\delta_{n o} \bar{\pi}_{i t-1}^{n o}+\delta_{o} \bar{\pi}_{i t-1}^{o}\right) .
$$

Theoretically, we expect $\bar{\pi}_{t}^{n o}>\bar{\pi}_{t}^{o}$. If there is learning based on past earnings ('reinforcement' learning), then subjects should pursue the optimal information decision and avoid the inferior one. In that case we expect $\delta_{n o}>0$ and $\delta_{o}<0$. However, subjects might during the course of the experiment come to understand that the strategic commitment of not seeing the Proposer's demand is optimal, irrespective of their past earnings experience. In that case $\delta_{T}>0$ should hold.

Table 2 presents the results of the panel probit estimations for treatment C. ${ }^{4}$ The estimated coefficients of model (2) are reported in the first column. All are significantly positive, as expected. The probability of making the predicted information decision increases over time and it is also positively related to having made this information decision in the previous period. This is evidence for learning at the individual level.

Model (3) is estimated in the table's second column. Only the estimate of $\delta_{n o}$ is significantly positive. The period coefficient $\delta_{T}$ and $\delta_{o}$ are insignificant at the $5 \%$ level. This is evidence of learning through positive earnings feedback. As a robustness check we also estimate the model with the each of the two experience variables individually (see the third and fourth column). The trend coefficient is once more positive. If we only include $\bar{\pi}_{i t-1}^{o}$ the estimated coefficient is as expected negative, and significantly different from zero at $p=0.065$.

Instead of recalling and being influenced by the previous payoffs from each information decision separately, subjects may consider the difference between the average payoffs, $\bar{\pi}_{i t-1}^{n o}-\bar{\pi}_{i t-1}^{o}$. To test this hypothesis we estimate the model with the constraint $\delta_{n o}=-\delta_{o}$; see the fifth column. The estimated coefficient of the difference between the average profits is significantly positive and so is the trend.

\footnotetext{
${ }^{4}$ All the models were also estimated with session dummies in order to control for session effects, but the dummies were never significant at the $5 \%$ level.
} 


\begin{tabular}{llllll}
\hline \hline & 1 & 2 & 3 & 4 & 5 \\
\hline cons & -.61 & -.80 & $-1.01^{*}$ & -.29 & .40 \\
& $(.38)$ & $(.61)$ & $(.42)$ & $(.68)$ & $(.44)$ \\
$n o_{i t-1}$ & $.79^{* *}$ & & & & \\
& $(.27)$ & & & & \\
PERIOD & $.17^{* *}$ & .08 & $.10^{* *}$ & $.23^{* *}$ & $.11^{*}$ \\
& $(.04)$ & $(.04)$ & $(.04)$ & $(.03)$ & $(.05)$ \\
$\bar{\pi}_{i t-1}^{n o}$ & & $.05^{* *}$ & $.05^{* *}$ & & \\
$\bar{\pi}_{i t-1}^{o}$ & & $(.01)$ & $(.01)$ & & \\
& & -.00 & & -.03 & \\
$\bar{\pi}_{i t-1}^{n o}-\bar{\pi}_{i t-1}^{o}$ & & $(.01)$ & & $(.02)$ & \\
Pseudo $R^{2}$ & .23 & & & & $.03^{* *}$ \\
AIC & 299.8 & 136.7 & 163.3 & 268.9 & 140.1 \\
$\#$ & 504 & 257 & 389 & 372 & 257 \\
\hline
\end{tabular}

Table 2: Responders' information decision over time and across treatments. Estimations of model (2) and (3) and their various mixtures with random effects for subjects. Standard errors in parentheses. AIC: Akaike Information Criterion. ${ }^{* *} 1 \%,{ }^{*} 5 \%$

Result 1. There is strong evidence that Responders in treatment UC make the predicted strategic commitment of seeing the Proposer's demand, and that Responders in treatment $C$ learn to make the predicted strategic commitment of not seeing the Proposer's demand. We fail to reject Hypothesis 1.

\subsection{Physical Timing Effects}

The difference in physical timing between the UC and the NC games could make the Responder more likely to avoid information about the Proposer's demand in the UC than in treatment NC. Figure 1 shows that on average and especially in the earlier periods fewer Responders see the Proposer's demand in the UC than in treatment NC. Over time however behavior in treatments UC and NC become indistinguishable. Indeed, based on the estimation of model (1) in the previous section, the joint hypothesis $\beta_{U C}=\beta_{N C}$ and $\beta_{T U C}=\beta_{T N C}$ cannot be rejected (Wald test, $p=0.631$ ). We find no evidence in favour of a physical timing effect.

Result 2. There is no evidence of a persistent physical timing effect. We fail to reject Hypothesis 2.

The results from the existing experimental literature can help explain why there is no persistent physical timing effect in our experiment. Our bargaining game is sequential and asymmetric, so physical timing cannot work as a symmetry breaker, as in Huck and Müller (2005). Moreover, since the Responder in all treatments makes his demand after the Proposer, the Responder is in a relatively weak position, as the Responder in the Ultimatum game (of course, due to reciprocity and fairness concerns they in practice get a higher payoff than what is theoretically predicted). The fact that the Responder in treatment UC makes his information decision before the Proposer makes 
his demand can not, according to the data, compensate for this weakness. Finally, any Responder strategy of not seeing the Proposer's demand is weakly dominated and, as pointed out by Güth, Huck, and Rapoport (1998), this weakens any physical timing effect. See also the findings in Weber, Camerer, and Knez (2006) and Huck and Müller (2005).

\subsection{Demands}

Hypothesis 3A Figure 2 shows the Proposer's and the Responder's average demands in each period in treatment $\mathrm{C}$ conditional on whether or not the Responder decided to see the Proposer's demand. Figure 3 shows the relative frequency distributions of Proposer and Responder demands. In treatment $\mathrm{C}$ the Proposer demands 50 points in 343 out of the 382 cases $(89.8 \%)$ where the Responder decided not to see the Proposer's demand. The Responder demands 50 points in 337 out of the same 382 cases $(88.2 \%)$. Pooling all Proposer and Responder demands across periods, the Proposer and Responder on average demand 49.5 and 50.2 points respectively when the Responder avoids seeing the Proposer's demand. When the Responder decides to see the Proposer's demand, the average across-period Proposer and Responder demands are 63.3 and 42.8 , respectively.

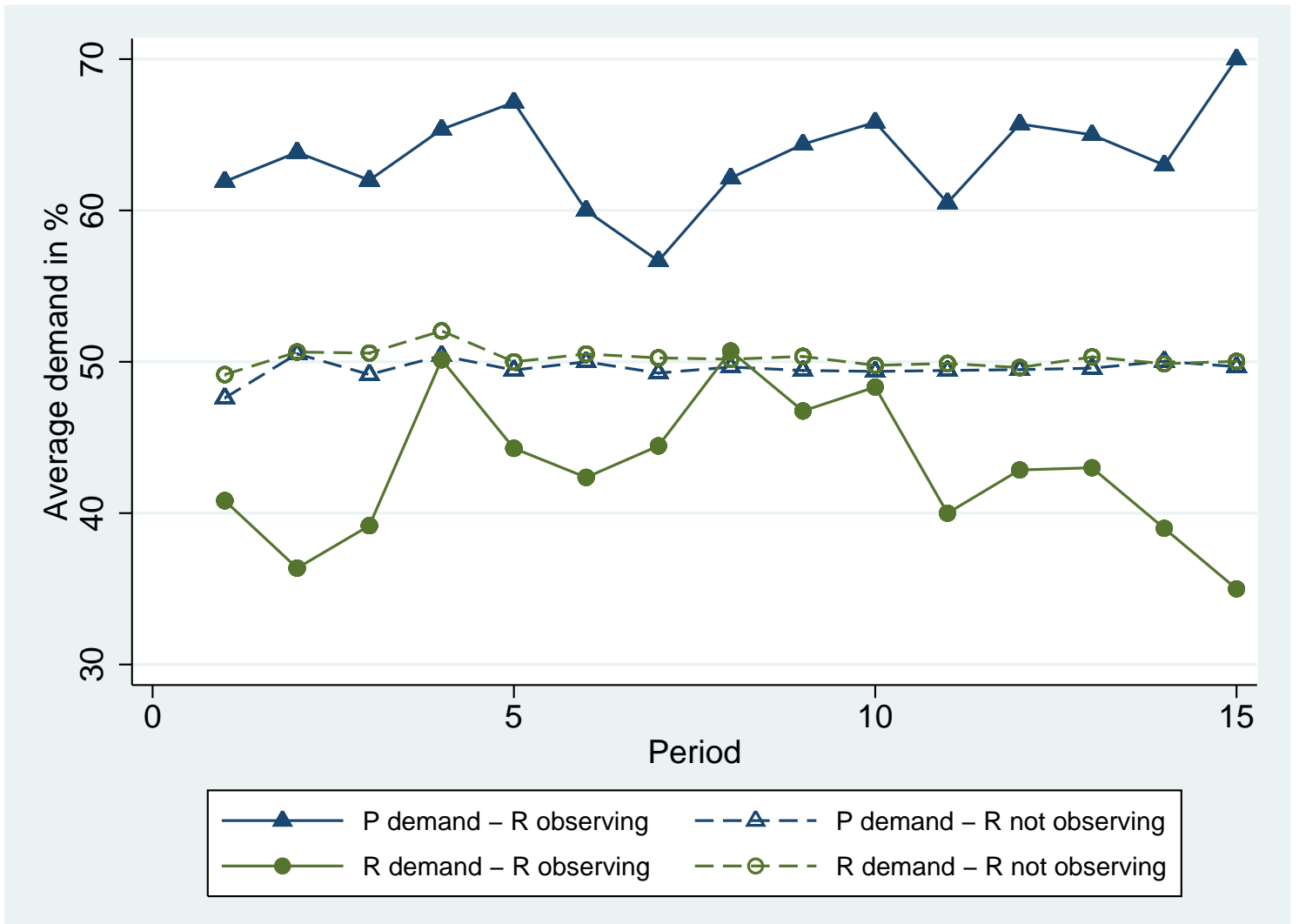

Figure 2: Average Proposer and Responder demands in treatment C, conditional on the Responder's information decision. $\mathrm{P}=$ Proposer, $\mathrm{R}=$ Responder.

In order to test for these and other differences we estimate a panel regression model: 

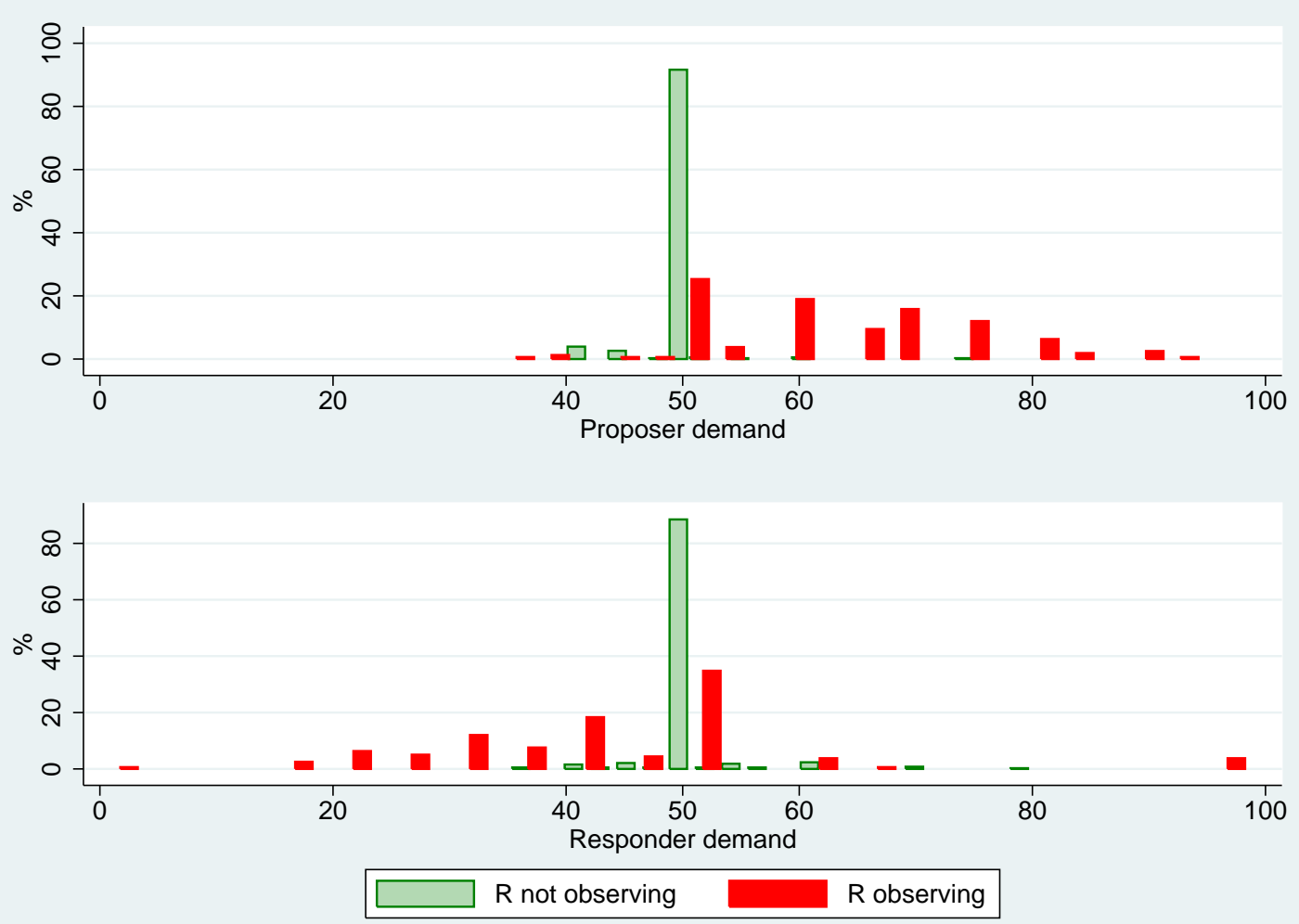

Figure 3: Relative frequency distributions of Proposer and Responder demands in treatment $\mathrm{C}$, conditional on the Responder's information decision. $\mathrm{R}=$ Responder.

$$
\begin{aligned}
x_{P, i t}=\alpha_{B M}+\alpha_{C} T_{C}+\alpha_{U C} T_{U C}+\alpha_{N C} T_{N C}+\alpha_{n o C} T_{C} n o_{i t}+ \\
\left(\alpha_{n o U C} T_{U C}+\alpha_{n o N C} T_{N C}\right) n o_{i t-1}+\varepsilon_{i t}, \\
x_{R, i t}=\alpha_{B M}+\alpha_{C} T_{C}+\alpha_{U C} T_{U C}+\alpha_{N C} T_{N C}+ \\
\left(\alpha_{n o C} T_{C}+\alpha_{n o U C} T_{U C}+\alpha_{n o N C} T_{N C}\right) n o_{i t}+\varepsilon_{i t},
\end{aligned}
$$

where $x_{k, i t}$ is the demand of player $i$ of type $k=P, R$ in period $t, T_{j}$ with $j=$ $C, U C, N C$ are treatment dummies (with $\mathrm{BM}$ as the reference treatment), and $n o_{i t}$ an indicator variable taking the value 1 when the Responder decides not to see the Proposer's demand. ${ }^{5}$ We use the lagged values of this indicator in the model for the Proposer in treatments UC and NC since in those treatments the Responder's

\footnotetext{
${ }^{5}$ We estimate a random effects model by GLS with robust standard errors adjusted for cluster effects in the sessions. The Breusch-Pagan LM test strongly rejects the null hypothesis of no random errors. The model was estimated with a linear time trend, but the trend coefficient is not significantly different from zero, which means that the demands do not change over time within the treatments. We also tested for autocorrelated error terms using the Wooldridge (2002) test for serial correlation in panel-data models, but did not find evidence of autocorrelation at any conventional significance level.
} 


\begin{tabular}{lllllllllll}
\hline \hline & & $\alpha_{B M}$ & $\alpha_{C}$ & $\alpha_{U C}$ & $\alpha_{N C}$ & $\alpha_{n o C}$ & $\alpha_{n o U C}$ & $\alpha_{n o N C}$ & $R^{2}$ & $\#$ \\
\hline \multirow{4}{*}{$x_{P}$} & coeff & 61.01 & 2.82 & -.16 & -.69 & -13.58 & -1.63 & -2.56 & .20 & 1778 \\
& $\sigma$ & 1.56 & 2.33 & 2.42 & 2.03 & 1.79 & 0.43 & 1.01 & & \\
& $\mathrm{p}$ & .000 & .226 & .948 & .733 & .000 & .000 & .012 & & \\
\hline \multirow{4}{*}{$x_{R}$} & coeff & 43.93 & -1.33 & .14 & 3.73 & 8.24 & 4.81 & 1.76 & .05 & 1905 \\
& $\sigma$ & 1.67 & 2.24 & 2.61 & 1.99 & 1.85 & .65 & 1.51 & & \\
& $\mathrm{p}$ & .000 & .553 & .957 & .061 & .000 & .000 & .245 & & \\
\hline
\end{tabular}

Table 3: Individual Proposer and Responder demands. Panel estimations of model (4) - (5) with random effects; robust standard errors adjusted for cluster effects.

information decision is not observable in the current period. Table 3 shows the results. The coefficient $\alpha_{n o C}$ is for both the Proposer and the Responder different from zero at any conventional significance level, indicating, as shown in Figure 2 and 3, that the Responder's information decision clearly impacts on both players' demand behavior in treatment C. Since $\alpha_{B M}+\alpha_{C}+\alpha_{n o C}$ is not significantly different from 50 (Wald tests, $p=0.722$ for $x_{P}$ and $p=0.417$ for $x_{R}$ ), players coordinate on the 50:50 split when the Responder avoids seeing the Proposers' demand.

Result 3. In treatment $C$ there is strong evidence that the 50:50 split is focal when the Responder does not see the Proposer's demand. We cannot reject Hypothesis 3 A.

Hypotheses 3B and 3C Since the dummy for treatment C, $\alpha_{C}$, is not significantly different from zero for both the Proposer and the Responder, we can state the following result.

Result 4. When in treatment $C$ the Responder decides to see the Proposer's demand, Proposer and Responder demands are equal to those in treatment BM. We cannot reject Hypothesis $3 B$.

Consider next Hypothesis 3C. The Proposer demands in treatments UC and NC are not significantly different, as $\alpha_{U C}=\alpha_{N C}$ cannot be rejected (Wald test, $p=.823$ ). Since both coefficients are not significantly different from zero there is also no difference from treatment BM. For the Responder, conditional on seeing the Proposer's demand, the demand in treatment $\mathrm{NC}$ is slightly higher than in treatment BM, but again there is no significant difference between NC and UC (Wald test, $p=0.104$ ). Comparing $\mathrm{C}$ with NC and UC, the only significant difference is that Responders in treatment NC make larger demands than those in treatment $\mathrm{C}, \alpha_{C}<\alpha_{N C}$ (Wald test, $p=0.004$ ). This difference can be attributed to the fact that when the Responder decides to be informed the Proposer knows this in $\mathrm{C}$ but not in treatment $\mathrm{NC}$; the Proposer then reacts by making larger demands in $\mathrm{C}$ than in treatment $\mathrm{NC}$ and the Responder reacts by demanding correspondingly less in $\mathrm{C}$ than in treatment NC. This is consistent with $\alpha_{C}>\alpha_{N C}$ for the Proposer, although the difference is not statistically significant.

Result 5. Proposer demands in treatments $U C$ and $N C$ equal those in treatment $C$ when the Responder sees the Proposer's demand. We can not reject Hypothesis $3 C$ for the Proposer. When the Responder sees the Proposer's demand, Responder demands are equal in the $U C$ and $B M$ and in treatments $U C$ and NC. Responder demands in treatment $N C$ are however larger than those in treatments $B M$ and $C$. We (weakly) reject Hypothesis $3 C$ for the Responder. 


\begin{tabular}{llllllllllll}
\hline \hline & $\gamma_{C}$ & $\gamma_{U C}$ & $\gamma_{N C}$ & $\gamma_{N O}$ & $\gamma_{N O U C}$ & $\gamma_{N O N C}$ & $\rho$ & $R^{2}$ & $D W_{o}$ & $D W_{t}$ & $\#$ \\
\hline coeff & 66.98 & 15.70 & 13.21 & 26.67 & -73.78 & -47.99 & .13 & .21 & 1.62 & 1.91 & 165 \\
$\sigma$ & 10.93 & 11.09 & 11.30 & 13.00 & 17.23 & 19.42 & & & & & \\
$\mathrm{p}$ & .000 & .187 & .269 & .052 & .002 & .033 & & & & & \\
\hline
\end{tabular}

Table 4: Average efficiency over treatments as a function of information avoidance. Estimation of model (6). $\rho$ denotes the coefficient of the first-order autoregressive error term; $D W_{o}$ is the Durbin-Watson statistic of the original model with $\rho=0 ; D W_{t}$ is the Durbin Watson statistic for the transformed model controlling for autocorrelation; $\sigma$ are robust standard errors adjusted for cluster effects.

The estimated values of the coefficients $\alpha_{n o U C}$ and $\alpha_{\text {noNC }}$ agree with intuition: When in treatments UC or NC the Proposer learns that the Responder with which he was matched in the previous period did not see the Proposers demand, the Proposer chooses a lower demand for the current period.

\subsection{Efficiency}

Let us consider efficiency, $e=\pi_{P}+\pi_{R}$. Similar to model (1) we regress ${ }^{6}$ the average level of efficiency in each session per period on treatment dummies $T_{i}$, where $i=U C$, $N C$, and on the fraction of Responders who decide not to observe the Proposer's demand in period $t, N O_{t}$ :

$$
e_{t}=\gamma_{C}+\gamma_{U C} T_{U C}+\gamma_{N C} T_{N C}+\left(\gamma_{N O}+\gamma_{N O U C} T_{U C}+\gamma_{N O N C} T_{N C}\right) N O_{t}+\varepsilon_{t}
$$

The Responder's decision not to observe the Proposers demand affects efficiency differently in treatment $\mathrm{C}$ and in treatments $\mathrm{UC}$ and NC. In treatment $\mathrm{C}$ the Responder's avoidance of information increases efficiency, since it almost always leads to the 50:50 split. When the Responder observes the Proposer's demand efficiency is lower since the Responder frequently decides to reject large Proposer demands by demanding more than the residual. These rejections seem to be driven by the same reciprocity and fairness concerns that are observed in the ultimatum game. In treatment BM about $22 \%$ of Responders demand more than the residual when the Proposer demands 65; if the Proposer demands 70,75 , or 80 , the percentages are 32,35 , and $70 \%$. In the treatment $\mathrm{C}$, when the Responder sees the Proposer's demand, the corresponding numbers are $20,37.5,50$, and $40 \%$.

In treatments UC and the NC a decision by the Responder not to see the Proposer's demand frequently leads to disagreement and hence zero efficiency since the Proposer, not knowing the Responder's information decision, typically demands more than half the surplus. On the other hand, when the Responder decides to observes the Proposer's demand efficiency is higher since the Responder then typically decides to demand the residual.

\footnotetext{
${ }^{6}$ Again, we use the Prais-Winston estimator and control for cluster effects using the sandwich estimator for the standard errors.
} 


\section{Discussion}

Our work is related to several other contributions. Using a sequential bargaining game Fischer, Güth, Müller, and Stiehler (2006) experimentally vary the probability that the second mover will observe the first mover's choice (see also Güth, Müller, and Spiegel, 2006). In their setup the probability with which the first mover's choice will be seen by the second mover is exogenous. Our paper is related to theirs in that it provides an answer to the following question: If the second mover could influence the probability of observing the first mover's demand, would the second mover understand that it would serve his or her interests best to set the probability equal to zero? Our theoretical answer is that the second mover should decide not to see the first mover's action when this decision is observed by the first mover, and our data show that subjects learn to understand this. ${ }^{7}$

Poulsen and Tan (2007) use the Ultimatum game to study if information acquisition interacts with fairness considerations. In their main treatment the Responder chooses a smallest acceptable offer (SAO) and the Proposer at the same time commits to seeing the SAO or not. The Proposer then makes an offer and the offer is accepted or rejected by the Responder according to the chosen SAO. In Poulsen and Tan's ultimatum game experiment it is the Proposer who makes an information decision about the Responder's acceptance threshold, while in the current sequential demand experiment it is the Responder who decides whether or not to see the Proposer's dermand. Moreover, there is an important difference in focus. Poulsen and Tan consider if players decide to obtain information that is predicted to be useful for a decision maker who seeks to maximize expected money earnings. They do not, however, consider if decision makers avoid harmful information. This is exactly what our $\mathrm{C}$ game addresses, and there is no analogue in Poulsen and Tan's experiment.

Our paper is also related to the literature on games with endogenous moves ('timing games'). See for example Datta Mago and Dechenaux (2009), Fonseca, Huck, and Normann (2005), and Fonseca, Müller, and Normann (2006). In these (mostly duopoly) games, the order of moves is endogenous. The literature seeks to understand whether sequential or simultaneous move games arise. In our design the order of moves is fixed and players instead decide on how much they would like to know when making a move. Nevertheless, from a game-theoretic point of view moving after another player but not knowing what the player did is the same as moving simultaneously. If one adopts this view our Commitment game corresponds to a timing game where one player moves first and decides between being an informed second mover or to move at the same time as the other player. ${ }^{8}$ Our data show that the predictions made by the theory of endogenous timing are, when sufficient learning has taken place, borne out for our bargaining experiment: A player learns that if the other player can condition

\footnotetext{
${ }^{7}$ In the light of the setup in Fischer et. al. (2006), it could be interesting to adapt our model such that the Responder, instead of making a yes/no information decision, decides on a probability of seeing the Proposer's demand. In the C game, the Proposer then observes the probability but not the realization. This version would correspond to the setup in Fischer et. al. (2006) if the observation probability in the latter model were chosen by the second mover and so endogenous. We conjecture that the second mover would then decide to set the probability equal to zero, as is done in the $\mathrm{C}$ game. We thank Werner Güth for indicating this relationship between the models.

${ }^{8}$ The formal structure of our Commitment game corresponds to a bargaining game with endogenous timing and with observable delay (Hamilton and Slutsky, 1990).
} 
his decision on the player's timing decision it is better to move at the same time as the other player than to wait and see what he or she did.

To which extent does our main finding, that players learn to optimally condition their strategic commitment on the strategic environment, generalize to other strategic situations? It is instructive to compare our findings with those from Fonseca, Müller, and Normann (2006). In their two-period duopoly quantity-setting game with observable delay (Hamilton and Slutsky, 1990) the unique equilibrium is that both players produce in period 1; in fact, moving in period 1 is a dominant strategy. In spite of this a substantial proportion of subjects decide to wait and move in period 2. The authors offer several explanations for their finding, such as inequality aversion and a preference for waiting, in order to resolve strategic uncertainty about the order of moves, although this is disadvantageous. ${ }^{9}$

It is not entirely clear why our results differ from those in Fonseca, Müller, and Normann's experiment. One explanation, pointed out by an anonymous reviewer, is that it can be easier for players to understand the value of commitment in some environments, such as our bargaining environment, than in other more complex situations. In a simple bargaining situation such as those captured by the Nash demand game a player will, after some learning, come to understand that deciding to move second makes one vulnerable to exploitation by first movers; in a duopoly game on the other hand players must not only learn this but must also at the same time learn the more complex underlying relationship between the players' output choices and profits. This hypothesis is consistent with the finding in Fonseca et. al. (2006) that the proportion of players who decide to wait falls but only very slowly. Understanding which strategic contexts are conducive for learning strategic commitments and which ones are not seems a fruitful area for future research.

\section{Conclusion}

Game theory predicts that rational and self-interested players optimally exploit any strategic commitment opportunity. Strategic commitments, such as 'burning the bridge', can however appear counterproductive or be too cognitively demanding for people to use in practice. We conducted a simple experiment using a simple bargaining game to see whether or not people commit to avoid harmful information. Our data show that the experimental subjects do after some time learn to make the predicted strategic commitment. Our results imply that the game theoretic intuition and modeling of strategic commitments seem, at least in our simple setup and once players has been given enough time to understand the situation, to have a solid empirical foundation.

\section{Appendix}

In this Appendix we prove Proposition 1. We first obtain a lemma that we then use to prove part $\mathrm{c}$ of Proposition 1. Fix some demand $y \in D$. In the UC game, consider

\footnotetext{
${ }^{9}$ For an analysis of inequality aversion, see Santos-Pinto (2008).
} 
the Responder strategy that sees the Proposer's demand, demands $X-x_{P}$ for any Proposer demand $x_{P}<X$, and demands $y$ if $x_{P}=X$. Denote this strategy $B R(y)$, and denote the set of all such strategies by $B R$.

Lemma 1. $i$. In the UC game, each Responder strategy in the set BR weakly dominates all the Responder strategies that are not in $B R$. ii. No strategy in $B R$ is weakly dominated.

Proof. Part i. The set of Responder strategies not in $B R$ can be partitioned into two groups: Those that do not see the Proposer's demand and makes some fixed demand, and those that see the Proposer's demand but do not play a best reply to some Proposer demand of less than the entire surplus. Consider a Responder strategy in the first group that makes some demand $x \in D$; denote this strategy $s_{x}$. Since $B R(y)$ by definition best replies to any Proposer demand of less than the entire surplus $X$, $B R(y)$ earns at least as high a payoff as $s_{x}$ against any such Proposer demand, and there is a non-empty set of Proposer demands against which $B R(y)$ earns a strictly higher payoff than $s_{x}$. Second, suppose the Proposer demands the entire surplus, $X$. Then $B R(y)$ earns the same as $s_{x}$, namely zero. This proves that $B R(y)$ weakly dominates $s_{x}$. Next, compare $B R(y)$ and any Responder strategy, denoted $s^{\prime}$, from the second group. That is, the Responder sees the Proposer's demand and there is at least one Proposer demand $x_{P}<X$ such that $s^{\prime}$ does not play a best reply to $x_{P}$. But then $B R(y)$ earns a strictly higher payoff against $x_{P}$ than does $s^{\prime}$, and since $B R(y)$ earns at least as high a payoff against any other Proposer demand, $B R(y)$ weakly dominates $s^{\prime}$, and part i of the lemma follows.

Part ii. Consider any strategy $B R(y) \in B R$. Suppose $B R$ is weakly dominated by some strategy. From part i of the lemma, any such strategy must be in $B R$. But all the strategies in BR earn the same payoff against any Proposer demand. Thus $B R(y)$ is not weakly dominated.

a. In any subgame-perfect Nash equilibrium of the BM game the Responder demands $x_{R}^{*}=X-x_{P}$ when observing that the Proposer demanded $x_{P}$ with $x_{P}<X$. If the Proposer demands the entire surplus, $x_{P}=X$, the Responder is indifferent between his feasible demands so any demand is optimal. It follows that the BM game has two pure-strategy subgame-perfect equilibria, described in the proposition.

b. Suppose first the Responder decides to see the Proposer's demand. The subgame that follows is identical to the BM game, so in any subgame-perfect equilibrium of the $\mathrm{C}$ game the Responder gets at most $\epsilon$ if he sees the Proposer's demand. Suppose the Responder decides not to see the Proposer's demand. From the assumption that the equal split then results it follows that the Responder in any subgame-perfect equilibrium of the overall game gets half of $X$ if he decides not to see the Proposer's demand. Since by assumption $X / 2>\epsilon$, it is optimal for the Responder to decide not to see the Proposer's demand. ${ }^{10}$

\footnotetext{
${ }^{10} \mathrm{~A}$ reviewer pointed out that the conclusion that the Responder will see the Proposer's demand will also hold if it were merely assumed that some Nash equilibrium giving the Responder at least $\epsilon$ is played in the subgame where the Responder decides not to see the Proposer's demand; the Responder strategy of not seeing the Proposer's demand and making the corresponding equilibrium demand would then weakly dominate any the Responder strategy that sees the Proposer's demand and that, on observing that the Proposer demands $X-\epsilon$ or $X$, demands the residual.
} 
c. There are many Nash equilibria in this game and in some the Responder does not see the Proposer's demand. ${ }^{11}$ However, Lemma 1 and the assumption that the Responder avoids weakly dominated strategies implies that the Responder uses a strategy in $B R$. This being common knowledge, the players' equilibrium demands are identical to those in the BM game described above.

d. As in the UC game, the Responder strategy of seeing the Proposer's demand and playing a best reply weakly dominates all other strategies. The proof is identical and hence omitted. Assuming once more that the Responder does not play weakly dominated strategies, the equilibrium demands for the $\mathrm{NC}$ game is the same as for the UC game.

\section{References}

Aghion, P. and Bolton, P. (1987). "Contracts as a Barrier to Entry", American Economic Review, 77 (3), 388-401.

Bagwell, K. (1995). "Commitment and Observability in Games", Games and Economic Behavior, 8 (2), 271-280.

Bensaid, B. and Gary-Bobo, R. (1993). "Commitment Value of Contracts Under Renegotiation Constraints", Econometrica, 61 (6), 1423-1429.

Ben-Porath, E. and Dekel, E. (1992). "Co-ordination and the potential for sacrifice", Journal of Economic Theory, 57 (1), 36-51.

Brocas, I. and Carrillo, J. (2007): "Influence through ignorance", The RAND Journal of Economics, 38 (4), 931 - 947.

Van Damme, E. (1989). "Stable Equilibria and Forward Induction", Journal of Economic Theory, 48 (2), 476-296.

Datta Mago, S. and Dechenaux, E. (2009). "Price leadership and firm size asymmetry: an experimental analysis", Experimental Economics, 12 (3), 289-317.

Fershtman, C. and Gneezy, U. (2001). "Strategic delegation: An experiment", RAND Journal of Economics, 32 (2), 352-368.

Fershtman, C. and Kalai, E. (1997). "Unobserved Delegation", International Economic Review, 38 (4), 763-774.

Fischer, S., Güth, W., Müller, W., and Stiehler, A. (2006). "From ultimatum to Nash bargaining: Theory and experimental evidence", Experimental Economics, 9 (1), 17-33.

Fischbacher, U. (2007). "z-Tree: Zurich Toolbox for Ready-made Economic Experiments", Experimental Economics, 10 (2), 171-178.

Fonseca, M., Huck, S., and Normann, H. (2005). "Playing Cournot although they shouldnt", Economic Theory, 25 (3), 669-677.

\footnotetext{
${ }^{11}$ For example, the following strategies are in a Nash equilibrium: The Responder does not see the Proposer's demand and demands one-half; the Proposer demands one-half.
} 
Fonseca, M., Müller, W., and Normann, H. (2006): "Endogenous timing in duopoly: experimental evidence", International Journal of Game Theory, 34, 443-456.

Greiner, B., (2004). "An Online Recruitment System for Economic Experiments", in: Kremer, K. and Macho, V. (eds.): Forschung und wissenschaftliches Rechnen 2003. GWDG Bericht 63, Göttingen: Gesellschaft für Wissenschaftliche Datenverarbeitung, 79-93.

Güth, W., Huck, S., and Rapoport, A. (1998). "The limitations of the positional order effect: Can it support silent threats and non-equilibrium behavior?", Journal of Economic Behavior and Organization, 34 (2), 313-325.

Güth, W., Müller, W., and Spiegel, Y. (2006). "Noisy leadership: an experimental approach", Games and Economic Behavior, 57 (1), 37-62.

Hamilton, J. and Slutsky, S. (1990). "Endogenous timing in duopoly games: Stackelberg or Cournot equilibria", Games and Economic Behavior, 2 (1), 2946.

Hauk, E. and Hurkens, S. (2001). "Secret information acquisition in Cournot markets", Economic Theory, 18 (3), 661-681.

Huck, S. and Müller, W. (2000). "Perfect versus imperfect observability - an experimental test of Bagwell's result", Games and Economic Behavior, 31 (2), 174190.

Huck, S. and Müller, W. (2005). "Burning money and (pseudo) first-mover advantages: an experimental study on forward induction", Games and Economic Behavior, 51 (1), 109-127.

Hurkens, S. and Vulkan, N. (2006): "Endogenous private information structures", European Economic Review, 50 (1), 35-54.

Loewenstein, G., Moore, D., and Weber, R. (2006). "Misperceiving the value of information in predicting the performance of others", Experimental Economics, 9 (3), 281-297.

Nash, J. (1953). "Two Person Cooperative Games", Econometrica, 21 (1), 128-140.

Poulsen, A. and Tan, J.H.W. (2007). "Information acquisition in the ultimatum game: An experimental study", Experimental Economics, 10 (4), 391-409.

Santos-Pinto, L. (2008). "Making sense of the experimental evidence on endogenous timing in duopoly markets", Journal of Economic Behavior and Organization, 68 (3-4), 657-666.

Schelling, T. (1960). The Strategy of Conflict. Cambridge, MA: Harvard University Press.

Weber, R., Camerer, C., and Knez, M. (2006). "Timing and Virtual Observability in Ultimatum Bargaining and "Weak Link" Coordination Games", Experimental Economics, 7 (1), 25-49.

Wooldridge, J. M. (2002). Econometric Analysis of Cross Section and Panel Data. Cambridge, MA: MIT Press. 